\title{
Quantitative Protection Factors for Common Masks and Face Coverings
}

\author{
David Leith ${ }^{1,2}$, Christian L'Orange ${ }^{1}$ and John Volckens ${ }^{1 *}$
}

(1) Department of Mechanical Engineering, Colorado State University, Fort Collins, CO

(2) Department of Environmental Sciences and Engineering, University of North Carolina at Chapel Hill, Chapel Hill, NC

\section{Supplementary Information}

Figure S1. Protection Factor for exhalation through masks for droplets with 5\% solids and for the residual particles left after these droplets have dried.... Page $S 2$

Figure S2 Relative Emission Factors for exhalation through masks for droplets with 5\% solids and for the residual particles left after these droplets have dried ....Page $S 3$

Table S1. Mask properties......Page S4

Figure S3. Typical size distribution for test aerosol by count and by mass.....Page S6

Mask Variability \& Model Sensitivity.....Page S7

Table S2. Properties of log-normal probability distributions used to represent variability in values of factors that affect PF and emissions.....Page $S 7$

Figure S4. Variability in protection factor during exhalation caused by variability in bypass alone (bypass only), fraction of time speaking alone (speech only), concentration when speaking alone (vocalization only), and by simultaneous variability in all three factors (combined effect)....Page $S 8$

Figure S5. Variability in protection factor during inhalation caused by variability in bypass alone (bypass only), fraction of time speaking alone (speech only), concentration when speaking alone (vocalization only), and by simultaneous variability in all three factors (combined effect)....Page $S 9$

Figure S6. Variability in relative emissions during exhalation caused by variability in bypass alone (bypass only), fraction of time speaking alone (speech only), concentration when speaking alone (vocalization only), and by simultaneous variability in all three factors (combined effect)....Page S10

Figure S7. Variability in relative emissions during inhalation caused by variability in bypass alone (bypass only), fraction of time speaking alone (speech only), concentration when speaking alone (vocalization only), and by simultaneous variability in all three factors (combined effect).....Page S11 
Figure S1. Protection Factor for exhalation through masks for droplets with 5\% solids and for the residual particles left after these droplets have dried. Table 1 lists values for $\mathrm{d}_{50}$ and $\sigma_{\mathrm{g}}$ for both size distributions.

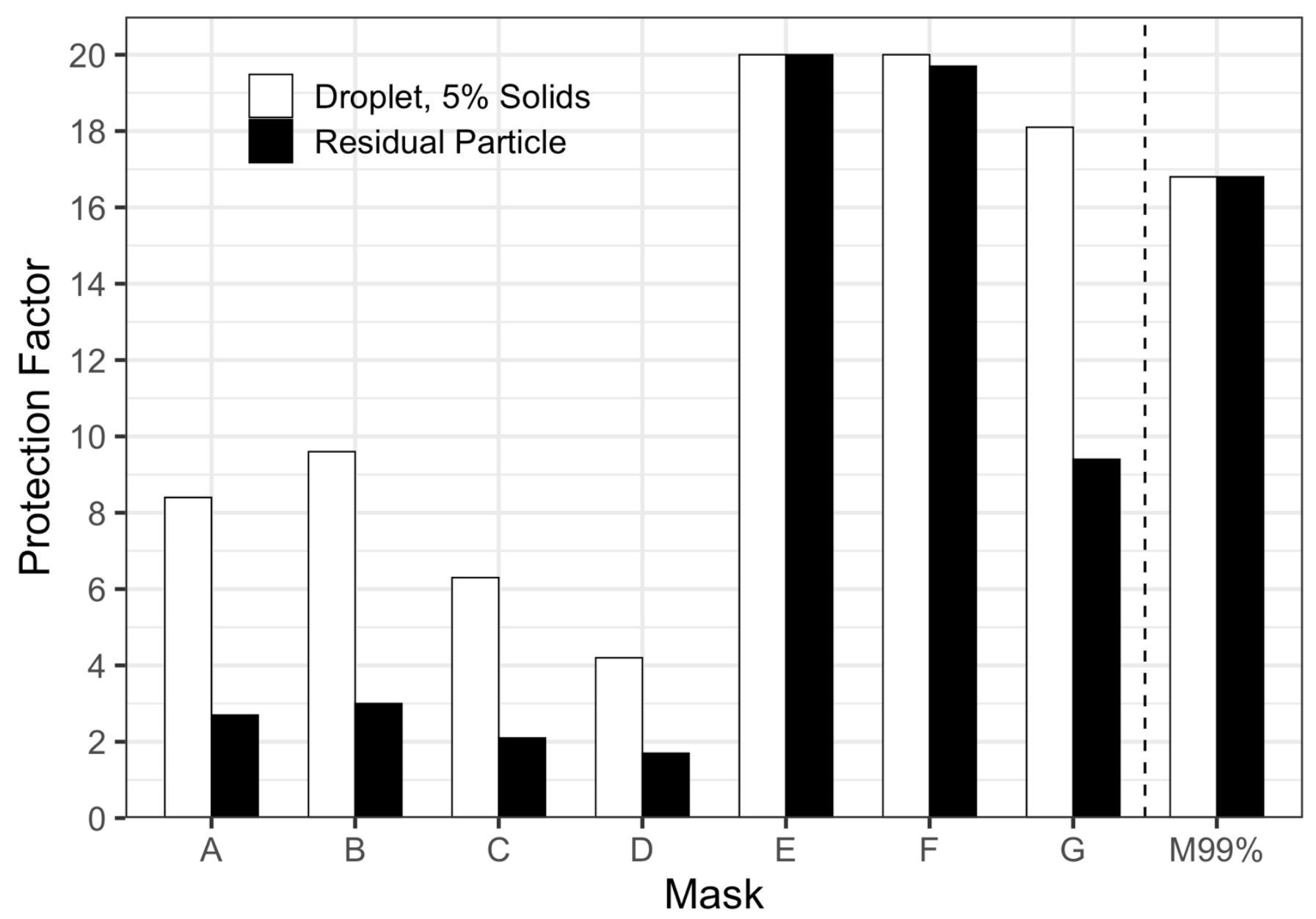


Figure S2 Relative Emission Factors for exhalation through masks for droplets with 5\% solids and for the residual particles left after these droplets have dried. Table 1 lists values for $\mathrm{d}_{50}$ and $\sigma_{\mathrm{g}}$ for both size distributions.

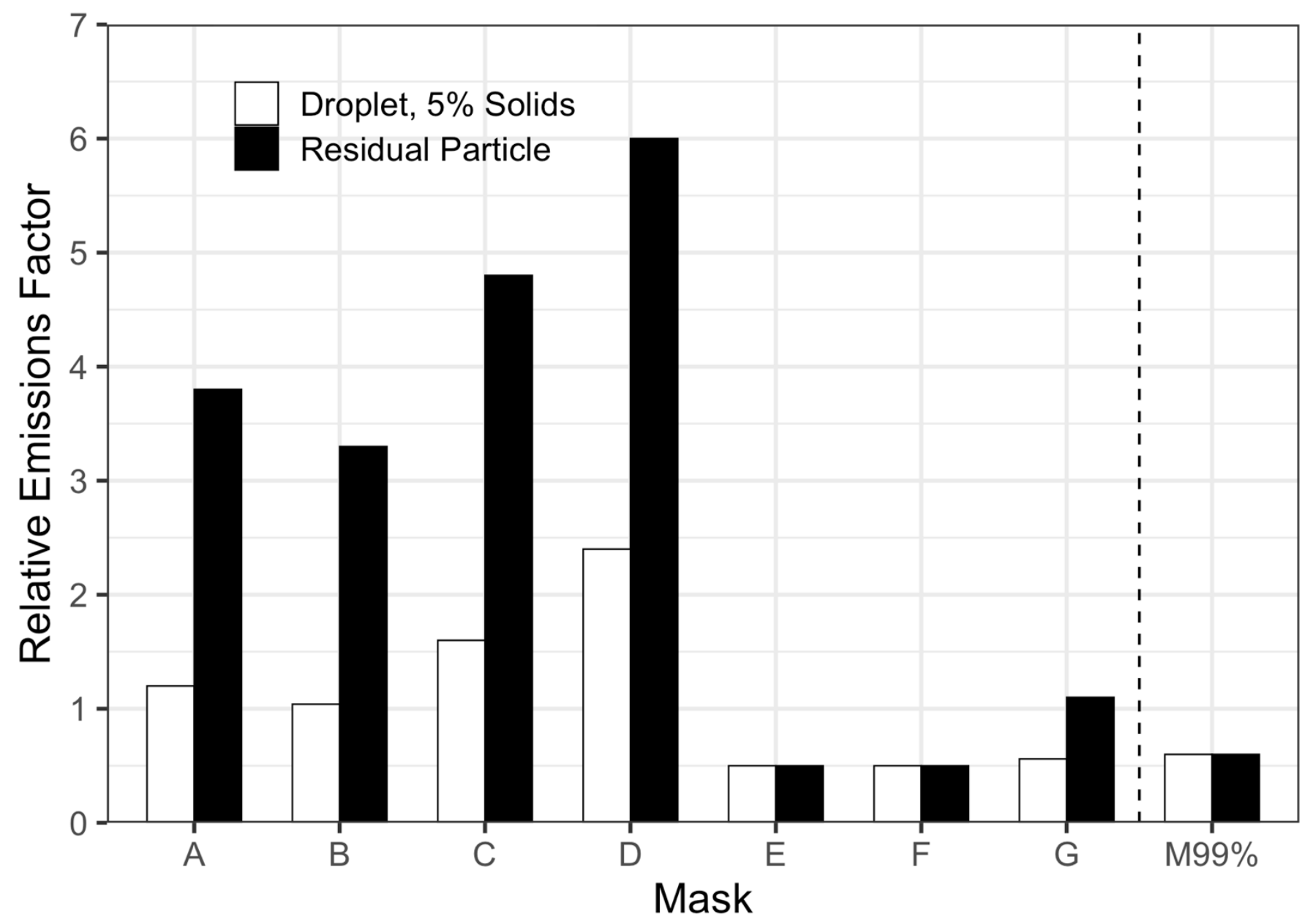


Table S1. Mask properties. "Outer" is mask side away from the wearer, "Inner" is mask side next to the wearer. df is fiber diameter; tpc is sum of thread count per $\mathrm{cm}$ in the warp and fill directions.

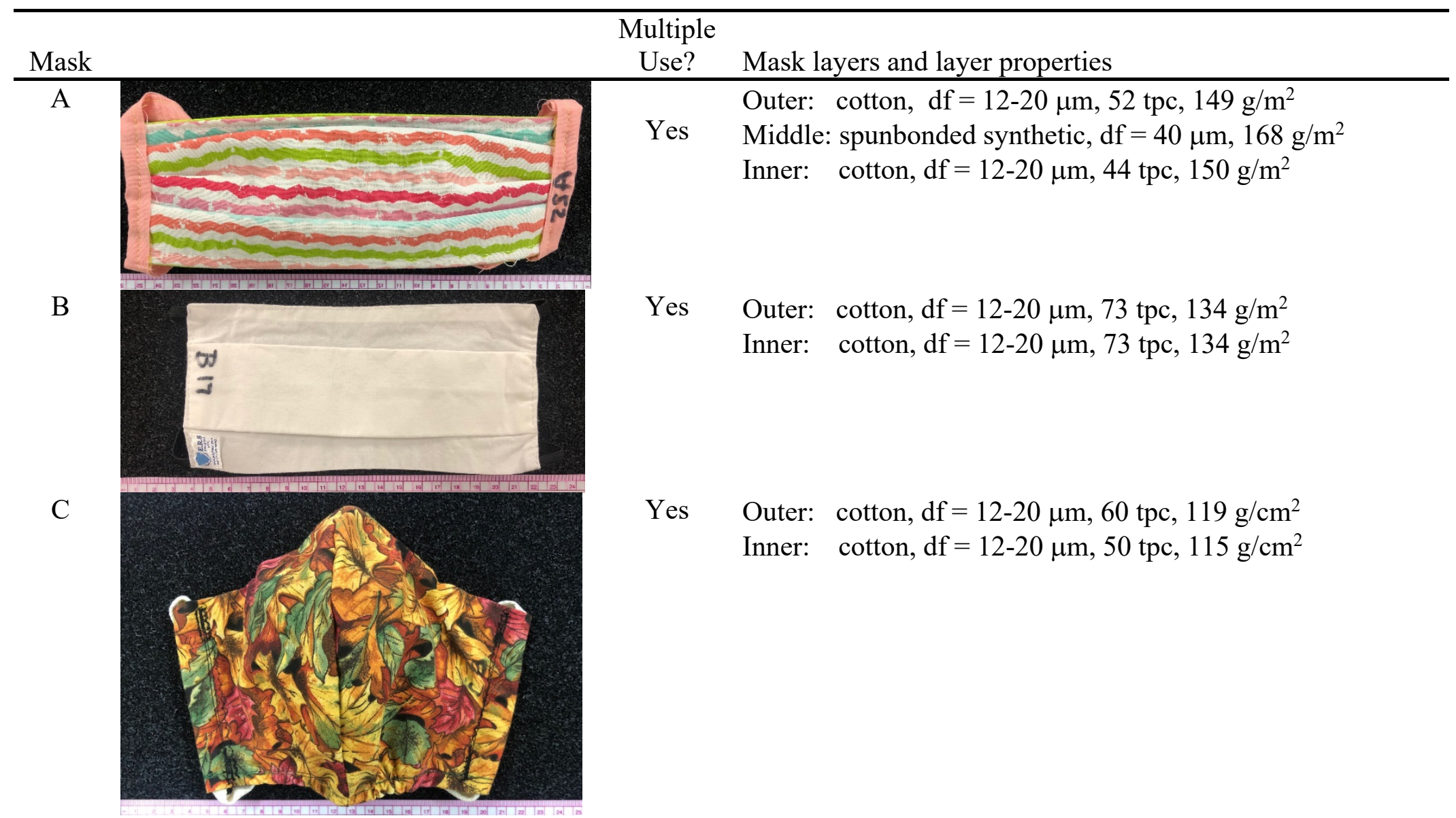


$\mathrm{D}$

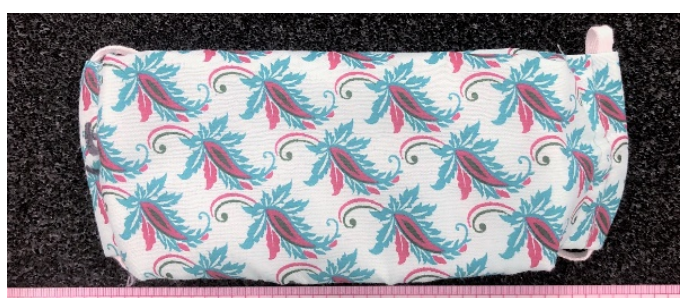

E

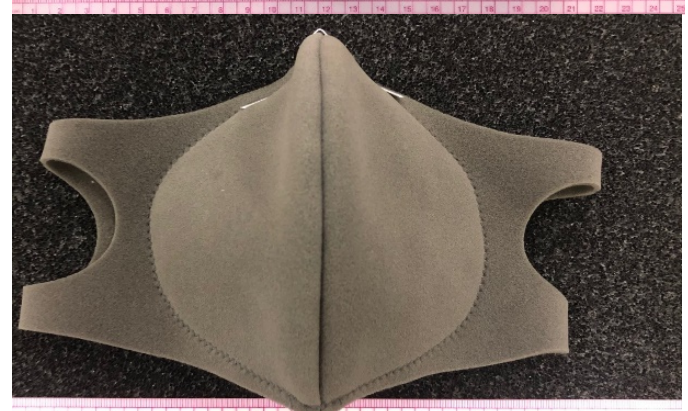

F

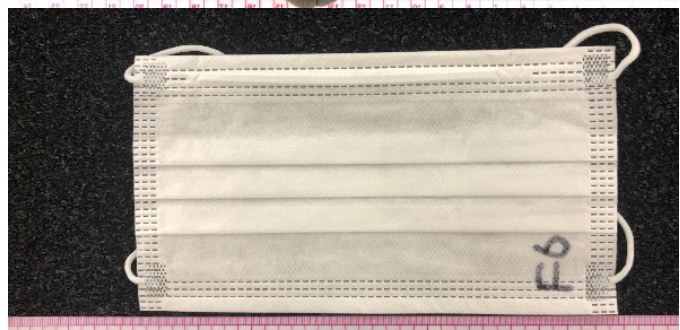

G

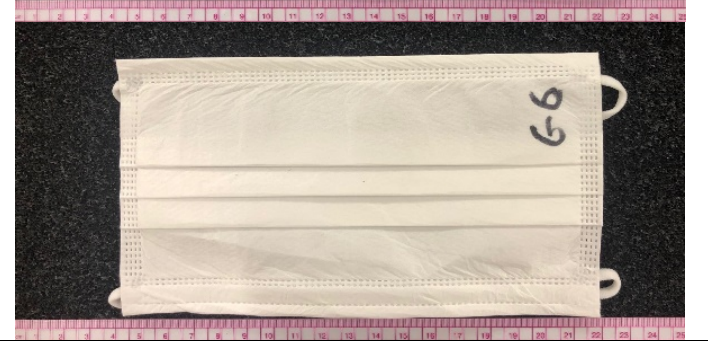

Outer: cotton, $\mathrm{df}=12-20 \mu \mathrm{m}, 54 \mathrm{tpc}, 68 \mathrm{~g} / \mathrm{m}^{2}$

Middle: spunbonded synthetic, $\mathrm{df}=40 \mu \mathrm{m}, 58 \mathrm{~g} / \mathrm{m}^{2}$

Inmer: cotton, $\mathrm{df}=12-20 \mu \mathrm{m}, 54 \mathrm{tpc}, 68 \mathrm{~g} / \mathrm{m}^{2}$

No Outer: porous foam, $150 \mu \mathrm{m}$ holes in foam, $127 \mathrm{~g} / \mathrm{m}^{2}$ Inner: synthetic nonwoven, $\mathrm{df}=2-7 \mu \mathrm{m}, 82 \mathrm{~g} / \mathrm{m}^{2}$, electrets?

Outer: spunbonded synthetic, $\mathrm{df}=20 \mu \mathrm{m}, 69 \mathrm{~g} / \mathrm{m}^{2}$

No Middle: synthetic fibers, $\mathrm{df}=3-5 \mu \mathrm{m}, 24 \mathrm{~g} / \mathrm{m}^{2}$, electrets?

Inner: $\quad$ spunbonded synthetic, $\mathrm{df}=20 \mu \mathrm{m}, 69 \mathrm{~g} / \mathrm{m}^{2}$

No Outer: single layer of synthetic fibers, $\mathrm{df}=4-7 \mu \mathrm{m}, 77 \mathrm{~g} / \mathrm{m}^{2}$ 
Figure S3. Typical size distribution for test aerosol by count and by mass. Error bars represent one standard deviation.

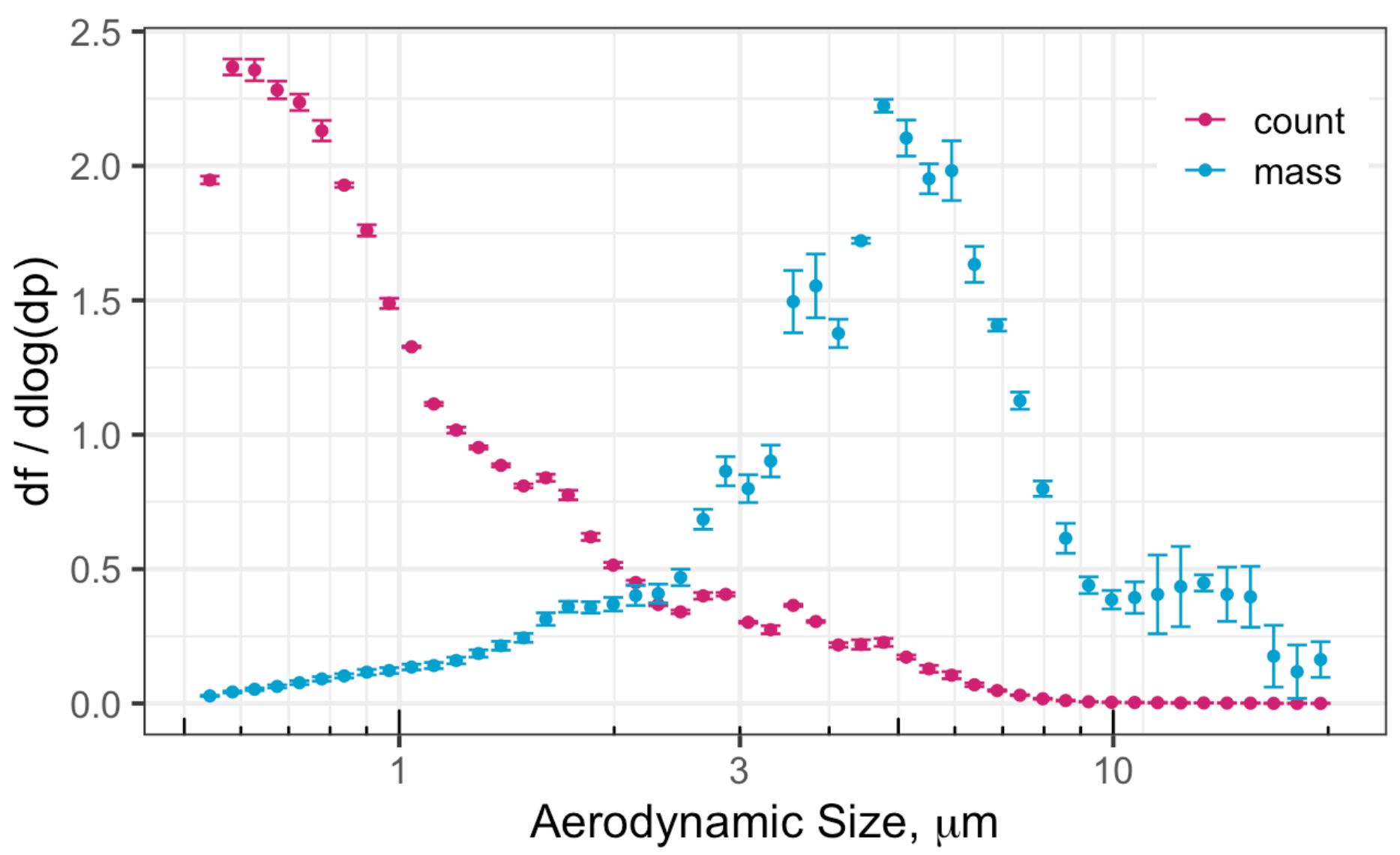




\section{MASK VARIABILITY}

We investigated the variability in protection factor and in emissions caused by variability in bypass alone, variability in fraction of time speaking alone and variability in level of vocalization alone, as well as the effect of variability in all three factors simultaneously. The variability for each factor was described by a log-normal probability distribution using published data whenever possible. The geometric mean of each factor's distribution, which is the $50^{\text {th }}$ percentile value of its cumulative probability distribution, was taken as its base case value in Table 1. The geometric standard deviation of each factor's distribution was estimated from its geometric mean and its cumulative $99^{\text {th }}$ percentile value, taken as an "upper" value based on previous studies. These values are in Table S2 for each factor's distribution, as are the associated "lower" values that have cumulative $1 \%$ probability.

Table S2. Properties of log-normal probability distributions used to represent variability in values of factors that affect PF and emissions.

\begin{tabular}{ccccc}
\hline $\begin{array}{c}\text { Factor } \\
\text { (independent } \\
\text { variable) }\end{array}$ & $\begin{array}{c}\text { Geometric mean } \\
(50 \%<\text { value }) \\
\text { from Table } 1\end{array}$ & $\begin{array}{c}\text { Upper Value } \\
(99 \%<\text { value })\end{array}$ & $\begin{array}{c}\text { Corresponding } \\
\text { geometric standard } \\
\text { deviation }\end{array}$ & $\begin{array}{c}\text { Corresponding } \\
\text { Lower Value } \\
(1 \%<\text { value })\end{array}$ \\
\hline $\begin{array}{c}\text { Fractional bypass } \\
\text { (Bypass) }\end{array}$ & 0.05 & 0.50 & 2.69 & 0.0098 \\
$\begin{array}{c}\text { Fraction of time } \\
\text { speaking } \\
\begin{array}{c}\text { (Speech) } \\
\text { Level of } \\
\text { vocaliazation } \\
\text { (Vocalization) }\end{array}\end{array}$ & 0.05 & 0.50 & 2.69 & 0.0098 \\
\hline
\end{tabular}

To investigate variability in PF or emissions due to variability in a given factor alone, a random number generator selected a value for a cumulative probability between 0 and 1 . This value was then used with the cumulative log-normal distribution function for that factor to determine the associated number of geometric standard deviations its value departed from its geometric mean, and from that calculation its value. This process was repeated to obtain a distribution of values for PF or emissions as a function of variability in that given factor.

To determine variability in PF or emissions for a single factor alone, the values for all other factors were set at their constant, base case values. For the factor under investigation, 100 random numbers were selected and the associated values for PF or emissions were determined for each mask for both exhalation and inhalation. Figures S4, S5, S6, and S7 show the resultant distributions of PF and emissions values for each mask, for the effect of variability in each factor alone.

To determine variability in PF or emissions for simultaneous variability in all three factors, separate calculations established a value for each factor, and these values were then used to determine PF and emissions. This process was repeated 100 times to establish the probability distributions for PF and emissions due to simultaneous variabilitiy in all three factors. Figures S4, S5, S6, and S7 show the resultant data as "Combined Effect;" these data are also shown in Figures 3 and 5. 
Figure S4. Variability in penetration factor during exhalation caused by variability in bypass alone (bypass only), fraction of time speaking alone (speech only), concentration when speaking alone (vocalization only), and by simultaneous variability in all three factors (combined effect).

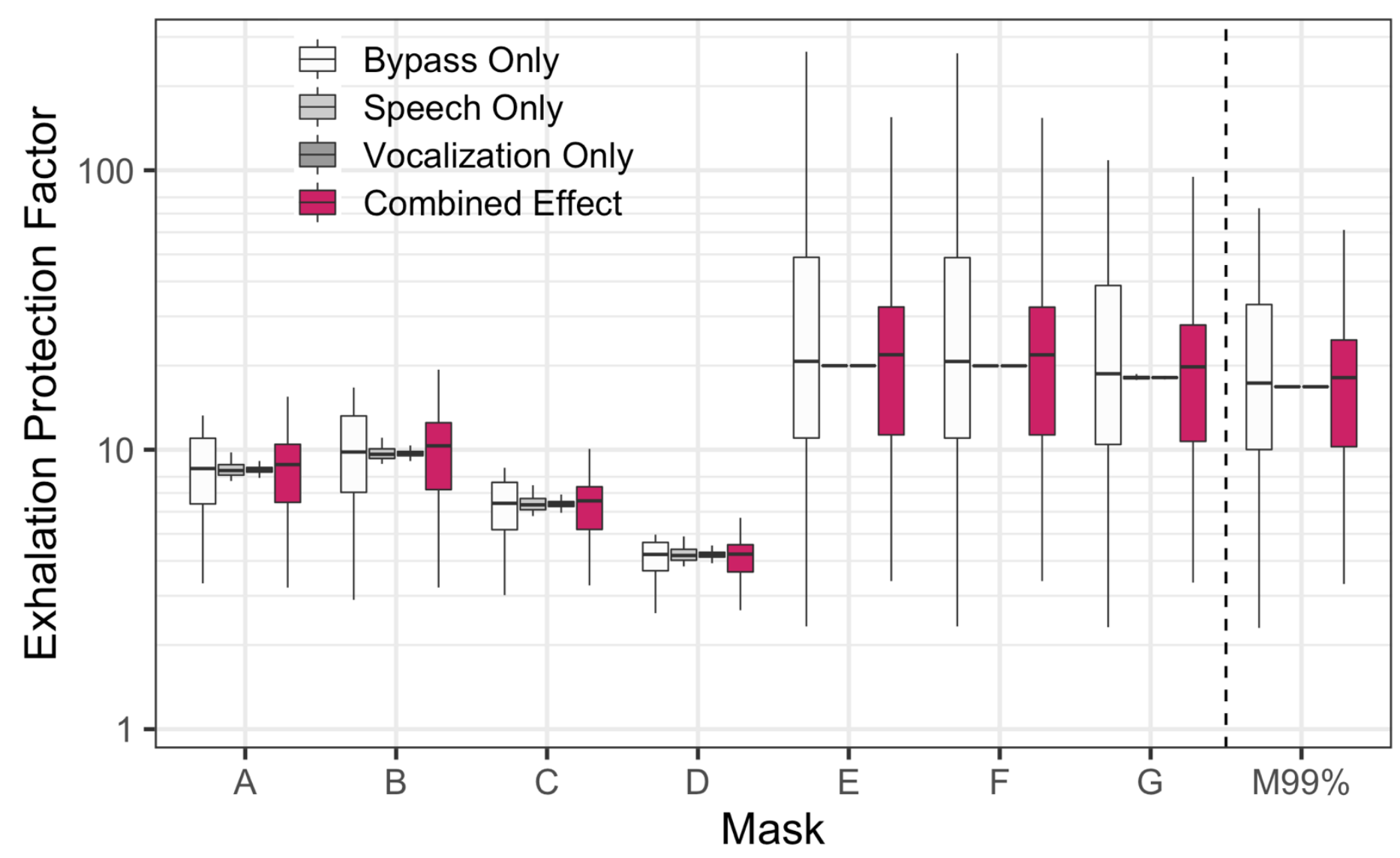


Figure S5. Variability in penetration factor during inhalation caused by variability in bypass alone (bypass only), fraction of time speaking alone (speech only), concentration when speaking alone (vocalization only), and by simultaneous variability in all three factors (combined effect).

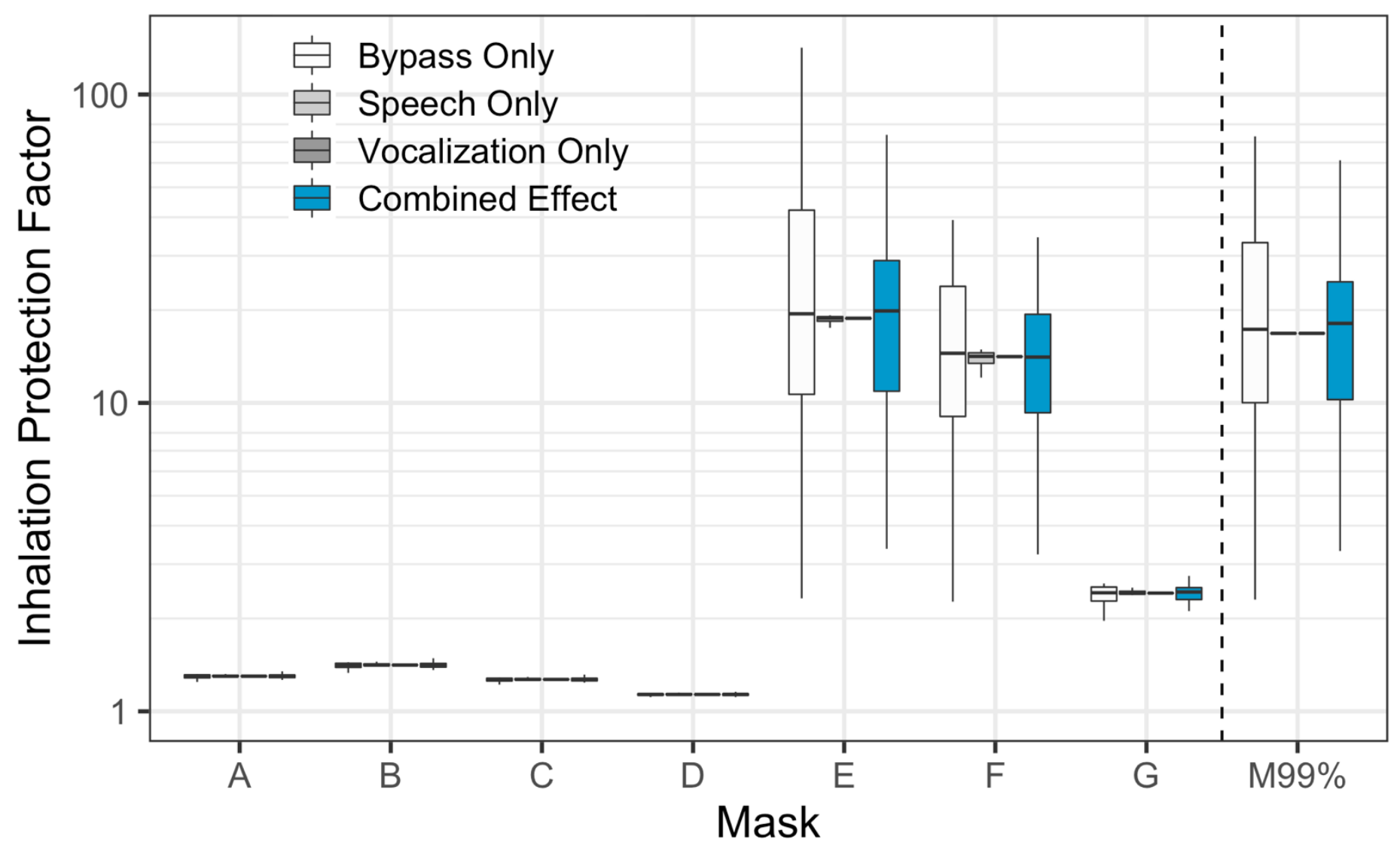


Figure S6. Variability in relative emissions during exhalation caused by variability in bypass alone (bypass only), fraction of time speaking alone (speech only), concentration when speaking alone (vocalization only), and by simultaneous variability in all three factors (combined effect).

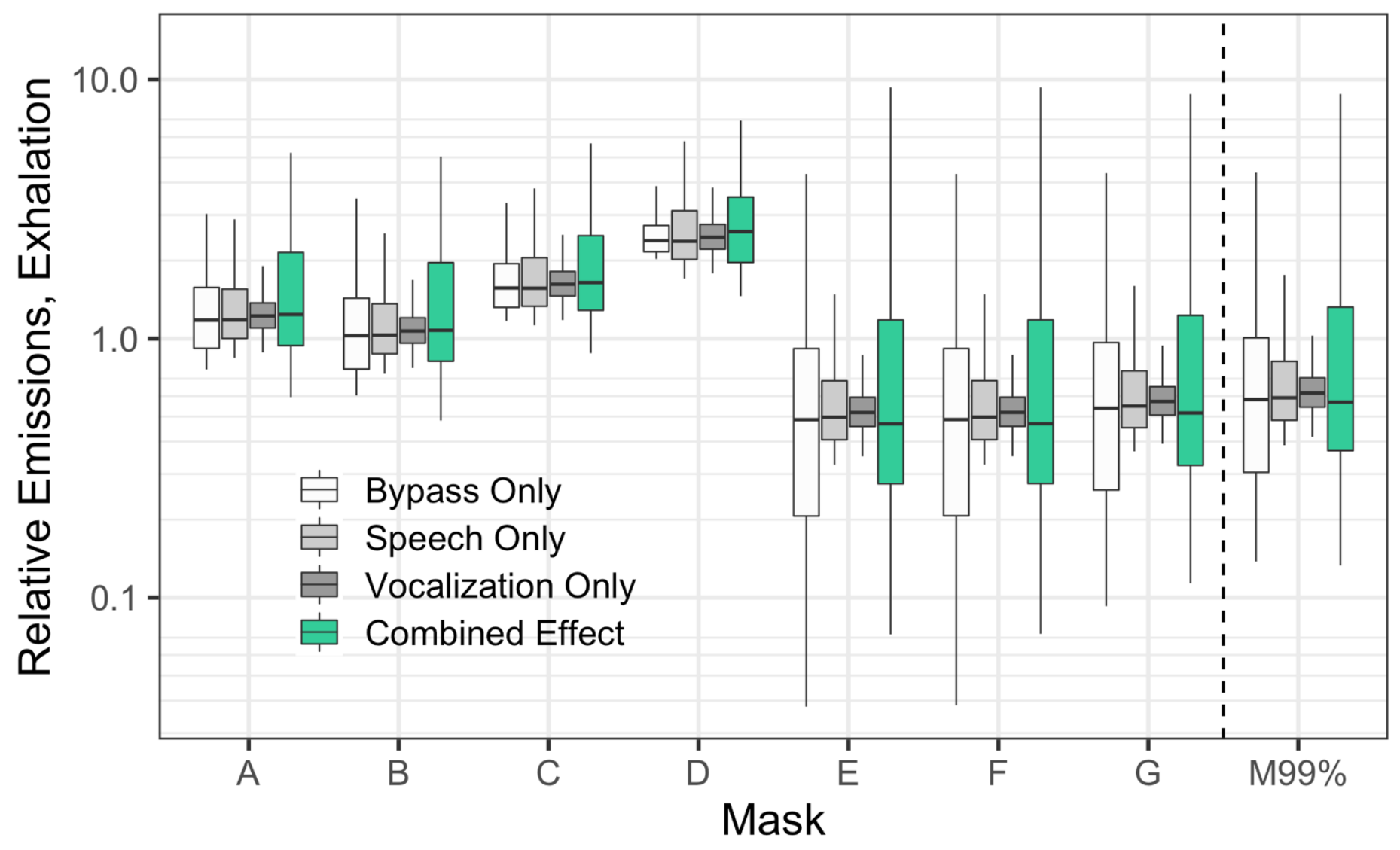


Figure S7. Variability in relative emissions during inhalation caused by variability in bypass alone (bypass only), fraction of time speaking alone (speech only), concentration when speaking alone (vocalization only), and by simultaneous variability in all three factors (combined effect).

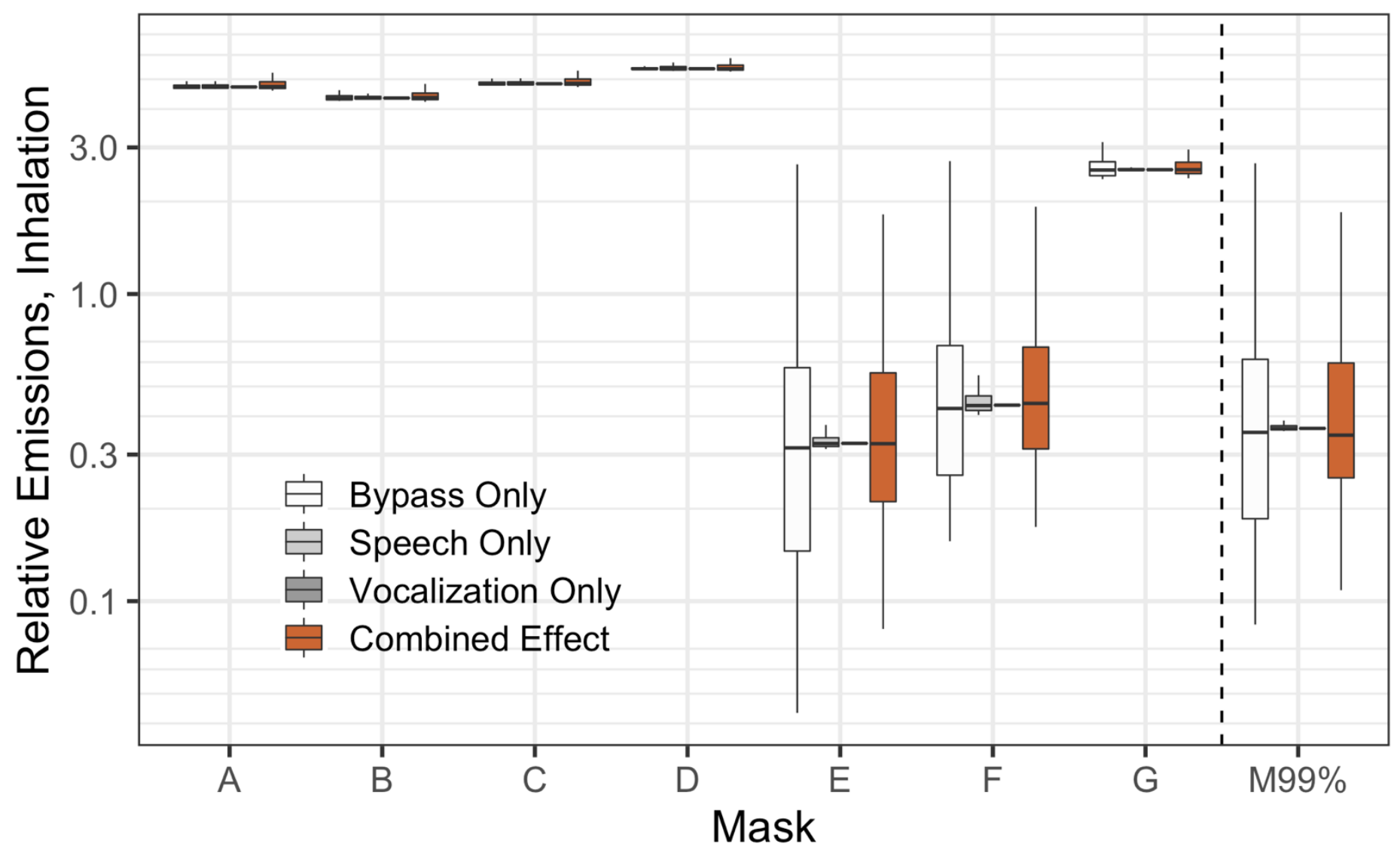

